\title{
Vibrational relaxation times in some hydrocarbons in the range $300-900^{\circ} \mathrm{K}$
}

\author{
James C. F. Wang and George S. Springer \\ Fluid Dynamics Laboratory, Department of Mechanical Engineering, The University of Michigan, Ann Arbor, Michigan 48105 \\ (Received 25 July 1973)

\begin{abstract}
Vibrational relaxation times were determined in methane, ethylene, acetylene, and cyclopropane in the temperature range $300-900^{\circ} \mathrm{K}$. The experiments were performed by measuring the absorption and dispersion of ultrasonic waves passing through the vapor. For all four vapors, the vibrational relaxation time $\tau_{v}$ (sec) was found to vary with temperature $T\left({ }^{\circ} \mathrm{K}\right)$ according to the relationship $\log _{10}\left(\tau_{v} P\right.$ ) $=a T^{T / 3}-b$, where $P$ is the pressure (atm) and $a$ and $b$ are constants. The results of the present measurements were also found to be in good agreement with existing data.
\end{abstract}

\section{INTRODUCTION}

The objective of this investigation was to determine experimentally vibrational relaxation times of various hydrocarbon vapors in the range $300-$ $900^{\circ} \mathrm{K}$. The experiments were performed with methane, ethylene, acetylene, and cyclopropane. For the former two vapors results are available up to $900{ }^{\circ} \mathrm{K},{ }^{1-5}$ but there is considerable scatter in the data to warrant further measurements. For acetylene and cyclopropane data exist only up to about 350 and $450{ }^{\circ} \mathrm{K}$, respectively. ${ }^{5,6}$

The relaxation times were determined by measuring the absorption of ultrasonic waves passing through the vapor. This technique has been employed successfully in the past for studying molecular transfer processes in gases both at room temperatures ${ }^{1-7}$ and, in recent years, also at higher temperatures. $4,8-10$

\section{GOVERNING EQUATIONS}

Consider an ultrasonic wave with circular frequency $\omega=2 \pi f$ propagating through a gas. The propagation constant (complex wavenumber) is given by ${ }^{1,11}$

$$
K=\omega / c-i \alpha,
$$

where $\alpha$ is the absorption coefficient and $c$ is the wave speed. In the absence of binary diffusion and chemical reaction, absorption is caused by viscothermal effects (viscosity and thermal conductivity) and by relaxation of the internal degrees of freedom of the molecules. When the absorption coefficient is small compared to the wavenumber $(\alpha \ll K)$ each of these sources of absorption may be treated separately, and the absorption coefficient may be expressed as ${ }^{1,11}$

$$
\alpha=\alpha_{c}+\alpha_{R} \text {. }
$$

$\alpha_{c}$ is the classical absorption coefficient ${ }^{1,11}$

$$
\alpha_{c}=\left(\frac{2 \pi^{2}}{\gamma \lambda}\right)\left[\frac{4}{3} \eta+\left(\frac{\gamma-1}{C_{p}}\right) k\right]\left(\frac{f}{P}\right) .
$$

In Eq. (3) $\eta$ is the kinematic viscosity of the gas, $k$ the thermal conductivity, $C_{p}$ the constant pressure specific heat, and $\gamma$ the specific heat ratio $\left(\gamma=C_{p} / C_{\nu}\right)$. The wavelength of the generated sound wave is $\lambda$, and $P$ is the pressure in the undisturbed medium. The absorption coefficient due to internal degrees of freedom is ${ }^{1,11}$

$$
\alpha_{R}=\frac{\pi}{\lambda}\left(\frac{C}{c_{0}}\right)^{2}\left(\frac{C_{p}^{\infty}}{C_{p}^{0}}-\frac{C_{v}^{\infty}}{C_{v}^{0}}\right) \frac{\omega \tau}{1+\left(C_{p}^{\infty} / C_{p}^{0}\right)^{2}(\omega \tau)^{2}} .
$$

$c / c_{0}$ is the sound dispersion ${ }^{1,11}$

$$
\frac{c}{c_{0}}=\left\{1-\frac{\left(C_{p}^{\infty} / C_{p}^{0}\right)\left(C_{p}^{\infty} / C_{p}^{0}-C_{v}^{\infty} / C_{v}^{0}\right)(\omega \tau)^{2}}{1+\left(C_{p}^{\infty} / C_{p}^{0}\right)^{2}(\omega \tau)^{2}}\right\}^{-1 / 2} .
$$

The subscripts and superscripts 0 and $\infty$ refer to conditions at equilibrium $(\omega=0)$ and at "frozen" flow $(\omega=\infty)$, respectively. $\tau$ is the relaxation time at constant pressure and temperature. Compressibility effects are not included in Eq. (5). To evaluate these effects, changes in the sound speed due to compressibility were calculated using the expression given by Carey et al. ${ }^{12}$ and the compressibility values presented by Tribus. ${ }^{13}$ For the conditions of the present experiments, compressibility did not appear to affect the sound speeds significantly.

It is seen from Eq. (4) that $\alpha_{R}$ reaches a maximum when

$$
\omega \tau=\left(C_{p}^{0} C_{v}^{0} / C_{p}^{\infty} C_{v}^{\infty}\right)^{1 / 2} .
$$

$\tau$ could thus be evaluated by measuring $\lambda_{R}$ for different values of the frequency and noting the value of $\omega$ (i.e., $\omega=\omega_{m}$ ) at which $\lambda_{R}$ reaches its maximum. Unfortunately, in practice it is difficult to vary $\omega$ over a sufficiently wide range. This experimental difficulty may be overcome by observing that the relaxation time is inversely proportional to pressure, at least when the pressure is sufficiently low so that collisions among three or more molecules may be neglected. Then, $\tau$ may be expressed as ${ }^{11}$

$$
\tau=B / P,
$$




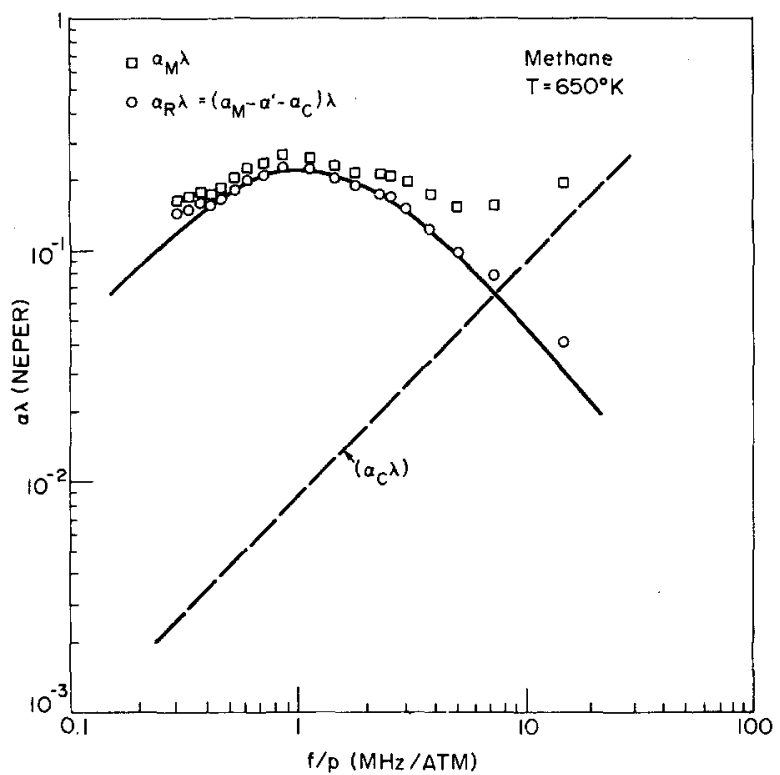

FIG. 1. The variation of the absorption coefficient with the frequency-pressure ratio. Methane at $650^{\circ} \mathrm{K}$. $\alpha_{c}$ calculated from Eq. (3). Solid line calculated from Eq. (8) with $B$ adjusted for best fit to data.

and Eqs. (4) and (6) become

$\lambda \alpha_{R}=\pi\left(\frac{C}{C_{0}}\right)^{2}\left(\frac{C_{p}^{\infty}}{C_{p}^{0}}-\frac{C_{v}^{\infty}}{C_{v}^{0}}\right) \frac{(2 \pi B)(f / P)}{1+\left(C_{p}^{\infty} / C_{p}^{0}\right)^{2}(2 \pi B)^{2}(f / P)^{2}}$,

$\frac{c}{c_{0}}=\left\{1-\frac{\left(C_{p}^{\infty} / C_{p}^{0}\right)\left(C_{p}^{\infty} / C_{p}^{0}-C_{v}^{\infty} / C_{v}^{0}\right)(2 \pi B)^{2}(f / P)^{2}}{1+\left(C_{p}^{\infty} / C_{p}^{0}\right)^{2}(2 \pi B)^{2}(f / P)^{2}}\right\}^{-1 / 2}$.

The proportionality constant $B$ is determined as ${ }^{(9)}$ follows. In the experiments $f$ is held constant and the pressure is varied providing a range of $f / P$ values. At each $f / P$ value $\alpha$ is determined experimentally. The absorption coefficient $\alpha_{R}$ is

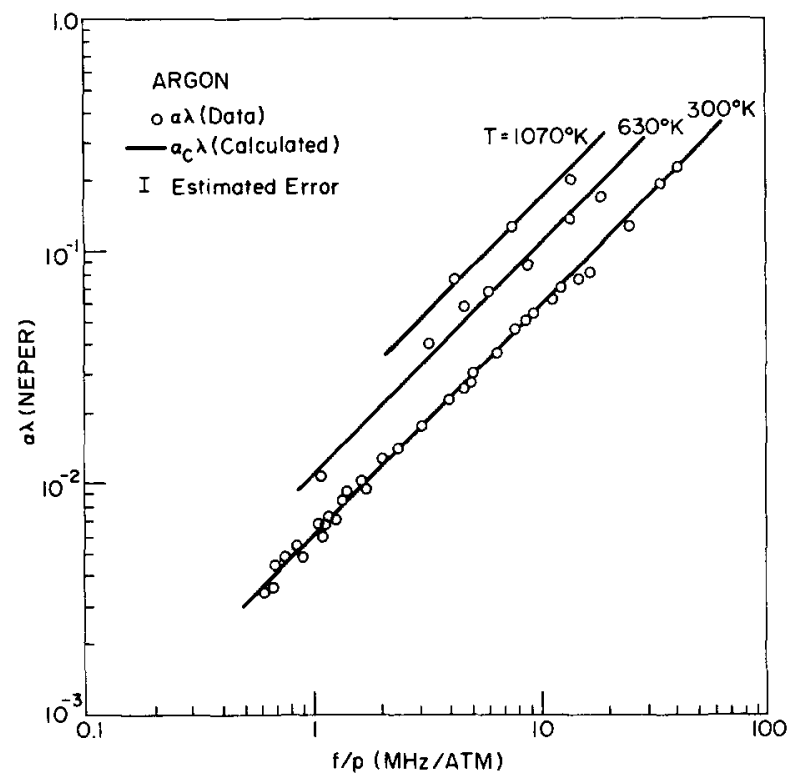

FIG. 3. The variation of the absorption coefficient with the frequency-pressure ratio. Argon. $\alpha_{c}$ calculated from Eq. (3).

evaluated by subtracting the appropriate value of $\alpha_{c}$ [calculated from Eq. (3)] from $\alpha$ [see Eq. (2)]. The $\alpha_{R}$ values thus obtained (or $\alpha_{R} \lambda$, since $\lambda$ is constant) are plotted against $f / P$. The construction of such a plot is illustrated in Fig. 1. In this figure $\alpha^{\prime}$ is a small correction to the data as explained in the next section. Further, $\alpha_{R} \lambda$ is calculated from Eqs. (8) and (9) for different values of $B$, and the value of $B$ is adjusted until the best match is found between the calculated $\alpha_{R} \lambda$ and the experimental data (solid line in Fig. 1).

Both vibrational and rotational relaxation may

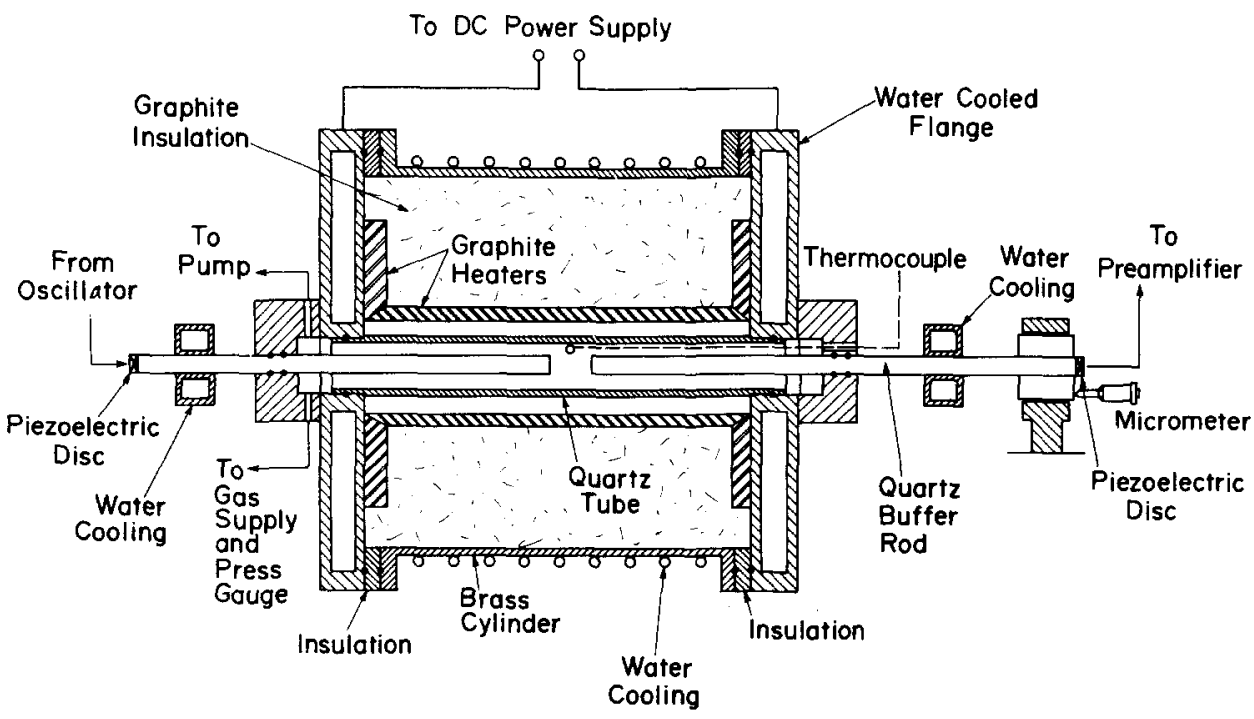

FIG. 2. Schematic of furnace and test section. 
contribute to $\tau$. Generally, these two internal degrees of freedom are excited independently and have different relaxation times. These relaxation times manifest themselves by separate maximums on the $\alpha_{R} \lambda$ versus $f / P$ plot. ${ }^{14}$ In our experiments only one maximum was observed, caused by vibrational relaxation. The maximum due to rotational relaxation would have occurred at higher $f / P$ values. ${ }^{3}$ The $f / P$ ratios needed to observe these second maximum points were outside the range of the apparatus.

\section{EXPERIMENTAL}

The experimental apparatus consisted of a furnace, an ultrasonic wave generator and signal detector, and a gas supply system. The furnace was constructed from a $23 \mathrm{~cm}$ diam, $0.3 \mathrm{~cm}$ thick and $25 \mathrm{~cm}$ long brass cylinder with a cooling water coil soldered around its outside (Fig. 2). Twenty $\mathrm{cm}$ diam and $5 \mathrm{~cm}$ thick brass flanges were bolted to each side of the cylinder. These flanges were hollow so that cooling water could be circulated through them. The flanges were separated from the cylinder by fiber glass insulation and Viton $O$ rings. A $5 \mathrm{~cm}$ diam hole was drilled at the center of each flange, which were covered by $7 \mathrm{~cm}$ thick brass plates. The buffer rods, gas supply lines, pressure and temperature gauges were introduced into the test section through these plates. The test section was a $5 \mathrm{~cm}$ diam and $0.2 \mathrm{~cm}$ thick fused quartz tube, mounted coaxially in the furnace and isolated from it by Viton $O$ rings. The test section was surrounded by a graphite tube $(7.5 \mathrm{~cm}$ diam, $0.3 \mathrm{~cm}$ thick) which served as the heating element. The two ends of this tube were tapered and were inserted into matching tapered holes provided in the centers of two $20 \mathrm{~cm}$ diam and $1 \mathrm{~cm}$ thick graphite plates. When the system was as sembled the heater tubes pressed these plates tightly against the brass flanges ensuring good electrical contact. The space between the graphite heater and the outer cylinder was filled with a graphite "blanket." Before each experiment this space was pumped down to about 0.2 torr and filled with argon or nitrogen. Power to the heater was provided by a germanium rectifier connected to the two flanges.

The ultrasonic wave was transmitted and received by $1.2 \mathrm{~cm}$ diam and $40 \mathrm{~cm}$ long fused quartz "buffer rods." These rods were inserted into the test section through the centers of the end plates and were positioned and sealed by double O-ring feedthroughs. The rod serving as the receiver could be moved in and out of the gas with a screw mechanism. This allowed the separation distance between the rods to be varied. The position of the movable rod was measured by a micrometer within $\sim 0.001 \mathrm{~cm}$. A $0.16 \mathrm{~cm}$ thick piezoelectric ceramic disk was attached to the "open air" ends of each

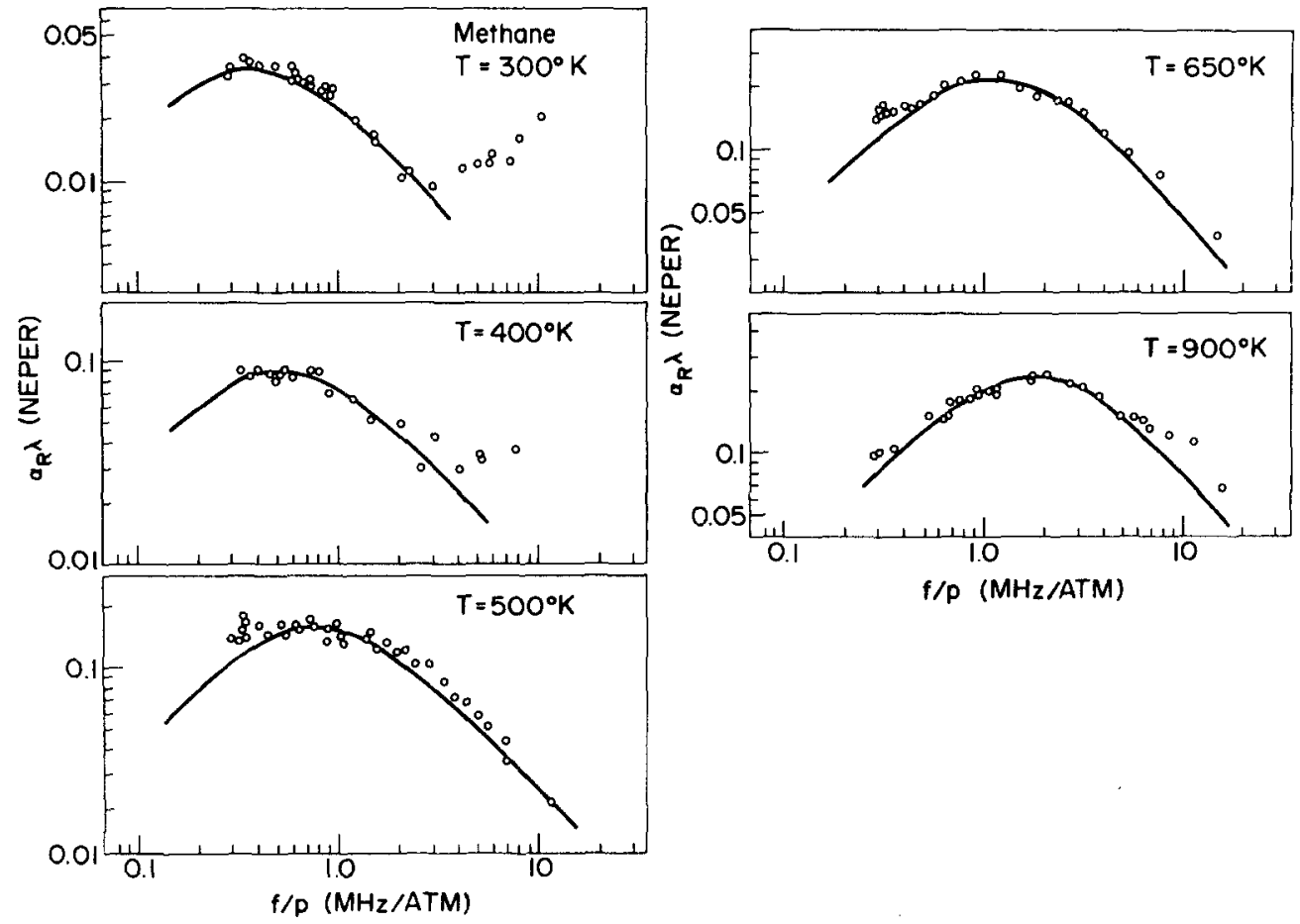

FIG. 4. The variation of the absorption coefficient with the frequency-pressure ratio in methane. OData. -Fit to data [calculated from Eqs. (8) and (9)]. 
buffer rod. These open ends were cooled by water, passing through a jacket enclosing the rods.

The ultrasonic wave was generated by an Arenberg high power pulsed oscillator. The output of the oscillator was fed to the piezoelectric disk through an impedance matching coil, which was provided to maximize the magnitude of the signal input to the disk. During the experiments the os cillator was operated at 0.5 and $1 \mathrm{MHz}$, with a pulse duration of $20 \mu \mathrm{sec}$. The frequency of the pulsed ultrasonic wave was measured by comparing (on an oscilloscope) the signal from the ultrasonic oscillator with a standard continuous sine wave produced by a beat frequency generator. When the two signals matched, the frequency of the signal from the beat generator was measured by a frequency counter. The signal from the receiver disk was fed into an Arenberg low frequency preamplifier. The output from this amplifier was displayed on a dual beam oscilloscope.

Before each experiment the test section and gas supply line were pumped down to $\sim 0.2$ torr. The test gas (Linde Research Grade 99.5\%- $99.9 \%$ pure) was then introduced into the test section through two bottles filled with $\mathrm{CaSO}_{4}$ and $\mathrm{P}_{2} \mathrm{O}_{5}$, respectively. The pressure was measured by a Heise gause and by a mercury manometer. The temperature inside the test section was monitored by a tungsten rhenium thermocouple located in the gas above the gap between the buffer rods.

The absorption coefficient and the speed of sound were measured by the differential path method, which has the advantage that the absolute distance

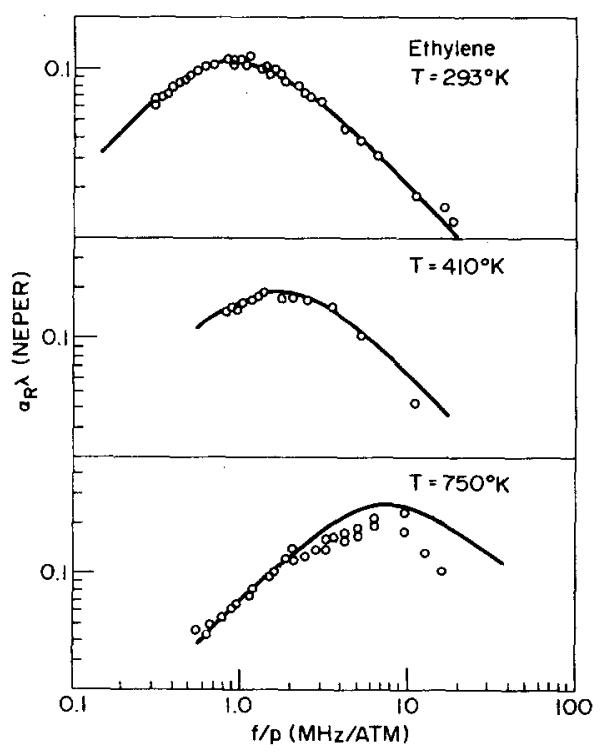

FIG. 5. The variation of the absorption coefficient with the frequency-pressure ratio in ethylene. O Data. - Fit to data [calculated from Eqs. (8) and (9)].

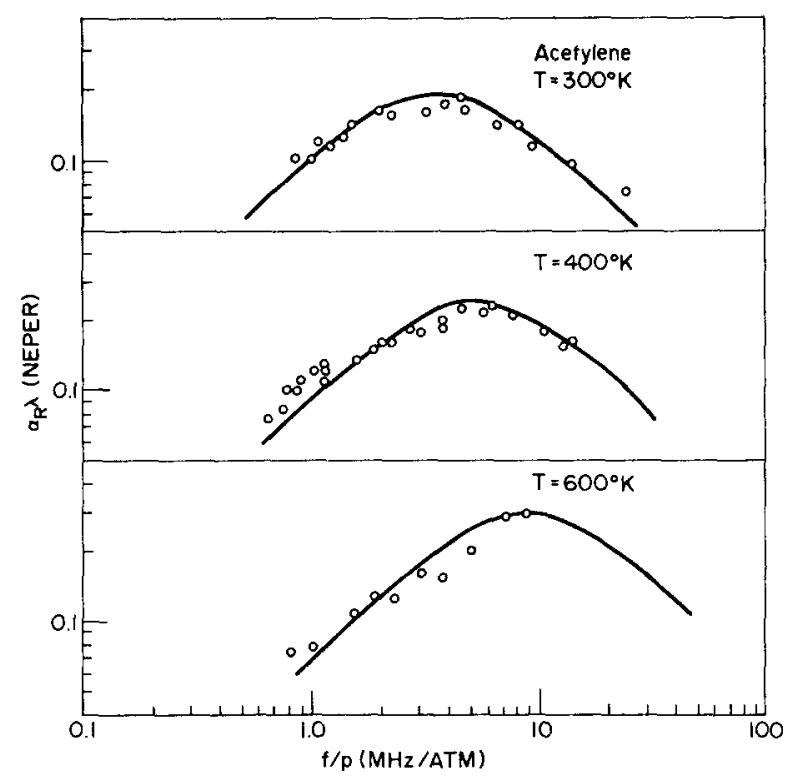

FIG. 6. The variation of the absorption coefficient with the frequency-pressure ratio in acetylene. O Data. -Fit to data [calculated from Eqs. (8) and (9)].

between the buffer rods need not be known accurate. ly. ${ }^{9}$ For a given $f / P$ ratio the distance $x$ between the buffer rods was varied, and for each value of $x$ the amplitude of the received wave $A$ was measured directly from the oscilloscope. By plotting $\log A$ versus $x$ one obtains a straight line, the slope of which yields the measured absorption coefficient $^{9}$

$$
\alpha_{M}=(d / d x)(\log A) .
$$

However, a correction must be applied to $\alpha_{M}$ due

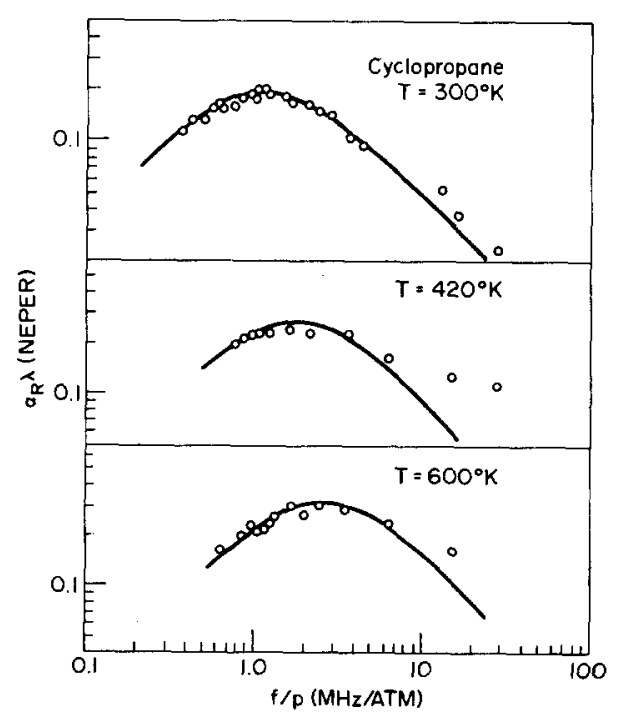

FIG. 7. The variation of the absorption coefficient with the frequency-pressure ratio in cyclopropane. 0 Data, -Fit to data [calculated from Eqs. (8) and (9)]. 
to effects introduced by the divergence of the sound beam. This correction is ${ }^{15}$

$$
\alpha^{\prime}=-(2 / L) \ln [1+(L / r) \phi],
$$

where $r$ is the radius of the piezoelectric disk, $L$ is the distance between the transmitter and receiver disks, and $\phi$ the angle of beam divergence

$$
\phi=\sin ^{-1}(0.61 \lambda / r) \text {. }
$$

Upon applying this correction the absorption coefficient becomes

$$
\alpha=\alpha_{M}+\alpha^{\prime} .
$$

In the present experiments $\alpha^{\prime}$ was usually less than $10 \%$ of $\alpha_{M}$.

The transit time $t$ of the wave between the transmitter and the receiver was also measured with a time delay trigger potentiometer built into the oscilloscope. The distance $x$ was plotted versus $t$ resulting in a straight line. The slope of this line is the wave speed $c$. The wavelength was calculated from this sound speed $(\lambda=c / f)$.

\section{RESULTS}

Prior to the experiment with the hydrocarbon vapors, measurements were performed with argon to test the usefulness of the apparatus. The mea- surements were conducted at three temperatures: 300,630 , and $1070^{\circ} \mathrm{K}$. The experimentally determined $\alpha_{R} \lambda$ values are represented by circles in Fig. 3. A detailed error analysis was made and the estimated magnitude of errors is also shown in Fig. 3. The classical absorption coefficient was calculated from Eq. (2) using the thermal conductivities and viscosities given by Hirschfelder et al. ${ }^{16}$ Since argon is incapable of either vibration or rotation, the calculated and measured $\alpha_{R} \lambda$ values should agree closely. Indeed, good agreement is evident between the experimental and calculated results, lending confidence to the operation of the apparatus. In the tests with argon the frequency-pressure ratios varied from 0.7 to 40 $\mathrm{MHz} / \mathrm{atm}$. In some of the subsequent experiments (most notably with methane) the $f / P$ ratios extended to $0.2 \mathrm{MHz} /$ atm. However, the error in the $f / P$ measurements is expected to be of the same order of magnitude at the lower $f / P$ values as at the higher ones. It is also noted that the major error in the data was caused not by uncertainties in the $f / P$ measurements, but by inaccuracies in the measurement of the wave speed.

The absorption coefficients measured in methane, ethylene, acetylene, and cyclopropane are presented in Figs. 4-7. In these figures the "best

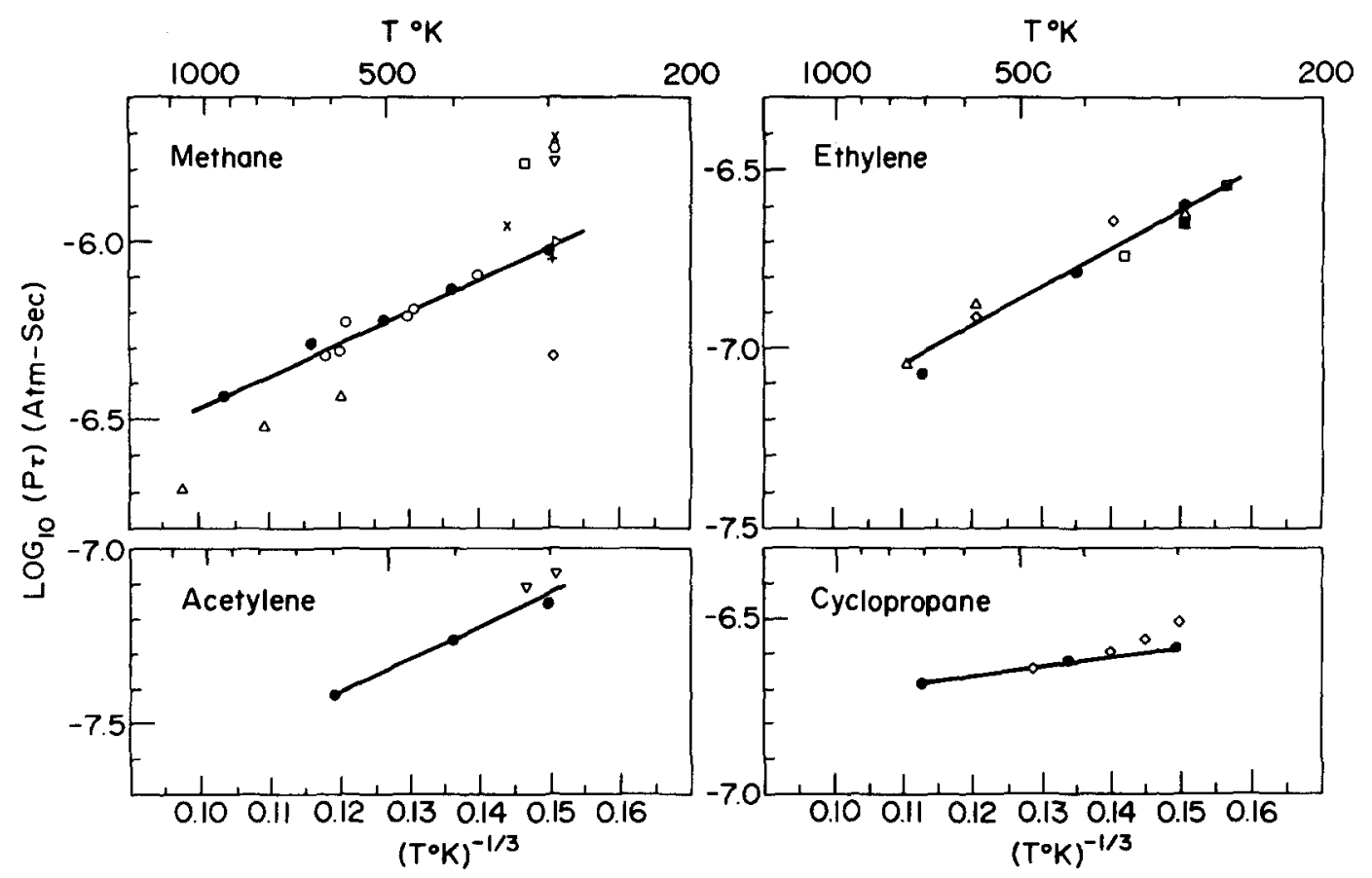

FIG. 8. The dependence of the vibrational relaxation time with temperature. - Present data. -Fit to present data [Eq. (14)]. Results of other investigators: Methane: OEucken and Aybar (1940); O Edmonds \& Lamb (1958); $\times$ Cottrell and Matheson (1962); $\square$ Cottrell and Martin (1957); $\nabla$ Cottrell and Day (1965); $\diamond$ Griffith (1950); + Gravitt, Whetstone, and Lagemann (1966); $\Delta$ Parker and Swope (1965); $\Delta$ Hill and Winter (1968). Ethylene: $\otimes$ R. Holmes and W. Tempest Proc. Phys. Soc. Lond. 78, 1502 (1961); $\Delta$ Hill and Winter (1968); $\diamond$ Corran, Lambert, Salter \& Warburton (1958). Acetylene: $\nabla$ Stretton (1965). Cyclopropane: $\diamond$ Corran, Lambert, Salter and Warburton (1958). 
fit" curves calculated from Eqs. (8) and (9) are also shown. The specific heat values used in the calculations are listed in Table 1. For each vapor a dominant single relaxation maximum was obtained within the experimentally attainable frequency/pressure range. A second maximum due to rotation would have occurred at higher $f / P$ values, which were outside the range of our apparatus. A trend in the data towards this second maximum was observed only with methane at 300 and $400{ }^{\circ} \mathrm{K}$ at the higher $f / P$ values (Fig. 4). In general, good match could be obtained between the measured and calculated absorption coefficients in the regions of interest, namely where the maximums occur. The worst discrepancy between the data and the calculated results is for ethylene (at $750{ }^{\circ} \mathrm{K}$ ) at high $f / P$ ratios. Here the data fall below the calculated values. This may be attributed either to a slight double relaxation or to dissociation of the gas. That the latter problem may have been present is supported by the evidence that a layer of carbon like deposition was found on the buffer rods inside the test section after these tests.

Vibrational relaxation times, calculated from the data in Figs. 4-7, are presented in Fig. 8. As can be seen, for all four vapors the vibrational relaxational time can be described by the expression

$$
\log _{10}\left(\tau_{v} P\right)=a T^{-1 / 3}-b
$$

where $\tau_{v}$ is the over-all relaxation time in seconds,

TABLE I. Specific heat values used in the calculations ${ }^{2}$ (cal $/{ }^{\circ} \mathrm{K}$, mole).

\begin{tabular}{lcccr}
\hline \multicolumn{1}{c}{ Gas } & Temp $\left({ }^{\circ} \mathrm{K}\right)$ & \multicolumn{1}{c}{$C_{b}^{0}$} & \multicolumn{1}{c}{$C_{v}^{0}$} & \multicolumn{1}{c}{$C_{p}^{0}-C_{p}^{\infty}$} \\
\hline $\mathrm{CH}_{4}$ & 300 & 8.552 & 6.566 & 0.608 \\
(from b) & 400 & 9.736 & 7.75 & 1.792 \\
& 500 & 11.133 & 9.147 & 3.189 \\
& 650 & 13.213 & 11.227 & 5.269 \\
& 900 & 16.21 & 14.224 & 8.266 \\
$\mathrm{C}_{2} \mathrm{H}_{4}$ & 293 & 10.38 & 8.394 & 2.436 \\
$($ from b) & 410 & 13.126 & 11.14 & 5.182 \\
& 750 & 19.98 & 17.994 & 12.036 \\
$\mathrm{C}_{2} \mathrm{H}_{2}$ & 300 & 10.532 & 8.546 & 3.581 \\
$($ from b) & 400 & 11.973 & 9.987 & 5.022 \\
& 600 & 13.728 & 11.742 & 6.777 \\
$\mathrm{C}_{6} \mathrm{H}_{6}$ & 300 & 13.44 & 11.454 & 5.496 \\
(from c) & 420 & 17.76 & 15.774 & 9.816 \\
& 600 & 24.24 & 22.254 & 16.296 \\
\hline (from & & &
\end{tabular}

${ }^{2}$ For $\mathrm{CH}_{4}, \mathrm{C}_{2} \mathrm{H}_{4}$, and $\mathrm{C}_{6} \mathrm{H}_{6}: C_{p}^{\infty}=7.944, C_{v}^{\infty}=5.958$. For $\mathrm{C}_{2} \mathrm{H}_{2}^{\infty}: C_{p}^{\infty}=6.951, C_{v}^{\infty}=4.965$.

${ }^{b}$ F. D. Rossini, et al., Selective Values of Physical and Thermodynamic Properties of Hydrocarbons (Carnegie, Carnegie Institute of Technology, Pittsburgh, 1953).

${ }^{\mathrm{c}} \mathrm{S}$. W. Benson, et al., Chem. Rev. 69, 279 (1969).
TABLE II. The constants $a$ and $b$ in Eq. (14).

\begin{tabular}{lcl}
\hline \hline Vapor & $a$ & \multicolumn{1}{c}{$b$} \\
\hline $\mathrm{CH}_{4}$ & 9.15 & 7.373 \\
$\mathrm{C}_{2} \mathrm{H}_{4}$ & 11.33 & 8.41 \\
$\mathrm{C}_{2} \mathrm{H}_{2}$ & 9.682 & 8.577 \\
$\mathrm{C}_{6} \mathrm{H}_{6}$ & 2.568 & 6.97 \\
\hline
\end{tabular}

$P$ is the pressure in atmospheres, and $T$ is the temperature in degrees Kelvin. The values of the constants $a$ and $b$ are listed in Table II. Thus, the present results follow the Landau-Teller expression, ${ }^{17}$ according to which $\log \left(\tau_{v} P\right)$ is proportional to $T^{-1 / 3}$.

For comparison, the existing experimental data are also shown in Fig. 8. For methane, the present data agree very well with the data of Eucken and Aybar, ${ }^{18}$ Parker and Swope, ${ }^{19}$ and Gravitt et al.${ }^{20}$ The results of Cottrell and his co-workers $\mathrm{s}^{21-23}$ and Edmonds and Lamb ${ }^{24}$ seem to be too high, while those of Griffith, ${ }^{25}$ and Hill and Winter ${ }^{4}$ appear to be too low. It is worth noting that at low temperatures the existing data differ by as much as $10 \%$ and at high temperatures by about $5 \%$. The present data fall in the middle of this "spread."

For ethylene, acetylene, and cyclopropane the results of the present measurements agree within about $1 \%$ of the existing data. Note, however, that only one set of measurements has yet been performed for acetylene (Stretton ${ }^{6}$ ) and one set for cyclopropane (Corran et al. ${ }^{5}$ ). These measurements extended only to 300 and $500{ }^{\circ} \mathrm{K}$ for acetylene and cyclopropane, respectively.

\section{ACKNOWLEDGMENTS}

The authors wish to thank C. Shen and P. Veenema for their help in the experiments. This work was supported by the National Science Foundation and by the Environmental Protection Agency.

${ }^{1}$ K. F. Herzfeld and T. A. Litovitz, Absorption and Dispersion of Ultrasonic Waves (Academic, New York, 1959).

${ }^{2}$ T. L. Cottrell and J. C. McCoubrey, Molecular Energy Transfer in Gases (Butterworths, London, 1959).

${ }^{3}$ J. L. Stretton, in Transfer and Storage of Energy by Molecules, edited by G. M. Burnett and A. M. North

(Wiley-Interscience, New York, 1969), Vol. 2, p. 58.

${ }^{4}$ G. L. Hill and T. G. Winter, J. Chem. Phys. 49, 440 (1968).

${ }^{5}$ P. G. Corran, J. D. Lambert, R. Salter, and B. Warburton, Proc. $\quad$ Soc. A 244, 212 (1958).

${ }^{6}$ J. L. Stretton, Trans. Faraday Soc. 61, 1053 (1965).

${ }^{7}$ R. Holmes, G. R. Jones, and N. Pusat, J. Chem. Phys. 41, 2512 (1964); Trans. Faraday Soc. 60, 1220 (1964).

${ }^{8}$ E. H. Carnevale, C. Carey, and G. Larson, J. Chem. Phys. 47, 2829 (1967).

${ }^{9}$ E. H. Carnevale, G. Larson, L. C. Lynnworth, C. Carey, M. Panaro, and T. Marshall, NASA Report No. 789 (1967). 
${ }^{10}$ T. G. Winter and G. L. Hill, J. Acoust. Soc. Am. 42, 848 (1967).

${ }^{1}$ R. T. Boyer and S. V. Letcher, Physical Ultrasonics (Academic, New York, 1969).

${ }^{12}$ C. Carey, E. H. Carnevale, S. Uva, and T. Marshall, "Experimental Determination of Gas Properties at High Temperatures and/or Pressures," AEDC-TR-69-78, Arnold Engineering Development Center (1969).

${ }^{13}$ M. Tribus, Thermostatics and Thermodynamics (Van Nostrand, Princeton, NJ, 1961).

${ }^{14}$ The rotational degrees of freedom differ from the vibrational ones in that they do not relax to equilibrium exponentially with a single time constant. However, even for rotational relaxation, generally, a single maximum is observed in the absorption versus frequency plot which provides a "rotational relaxation frequency." See Refs. 1-3.

${ }^{15}$ V. F. Nozdrev, The Use of Ultrasonics in Molecular Physics, translated by J. A. Cade, revised by E. R. Dobbs (MacMillan,
New York, 1965).

${ }^{16}$ J. O. Hirschfelder, C. F. Curtiss, and R. B. Bird, Molecular Theory of Gases and Liquids (Wiley, New York, 1954).

${ }^{17}$ L. Landau and E. Teller, Phys. Z. Sowjetunion 10, 34 (1936).

${ }^{18}$ A. Eucken and S. Aybar, Phys. Chem. 46B, 195 (1940).

${ }^{19}$ J. G. Parker and R. H. Swope, J. Chem. Phys. 43, 4427 (1965).

${ }^{20} \mathrm{~J}$. C. Gravitt, C. N. Whetstone, and R. T. Lagemann, J. Chem. Phys. 44, 70 (1966).

${ }^{21}$ T. L. Cottrell and A. J. Matheson, Trans. Faraday Soc. 58, 2336 (1962).

${ }^{22}$ T. L. Cottrell and P. E. Martin, Trans. Faraday Soc. 53, 1157 (1957).

${ }^{23}$ T. L. Cottrell and M. A. Day, J. Chem. Phys. 43, 1433 (1965).

${ }^{24}$ P. D. Edmonds and J. Lamb, Proc. Phys. Soc. Lond. 72, 940 (1958).

${ }^{25}$ W. Griffith, J. Appl. Phys. 21, 1319 (1950). 